Revue d'histoire de l'Amérique française

ZWB REVUE D.HISTOIRE DE L'AMÉRIQUE FRANÇAISE

\title{
Les sources privées de l'histoire coloniale dans le pays de Bigorre (1952-1957)
}

\section{Roger Massio}

Volume 12, numéro 3, décembre 1958

URI : https://id.erudit.org/iderudit/301922ar

DOI : https://doi.org/10.7202/301922ar

Aller au sommaire du numéro

Éditeur(s)

Institut d'histoire de l'Amérique française

ISSN

0035-2357 (imprimé)

1492-1383 (numérique)

Découvrir la revue

Citer cet article

Massio, R. (1958). Les sources privées de l'histoire coloniale dans le pays de Bigorre (1952-1957). Revue d'histoire de l'Amérique française, 12(3), 407-410. https://doi.org/10.7202/301922ar d'utilisation que vous pouvez consulter en ligne.

https://apropos.erudit.org/fr/usagers/politique-dutilisation/ 


\section{LES SOURCES PRIVÉES DE L'HISTOIRE COLONIALE DANS LE PAYS DE BIGORRE}

(1952-1957)

Notre intention reste la même que dans notre précédente chronique: ${ }^{1}$ présenter les différents papiers privés ayant trait à l'histoire coloniale que nous avons pu rencontrer ces dernières années, observer à travers ces découvertes les principaux problèmes de la France d'outre-mer au XVIIIème siècle.

\section{Les Papiers Lacaye et Laporte. ${ }^{2}-$}

Des papiers Lacaye demeure seul un testament, de Laporte également très peu de choses. On a deux aperçus rapides mais combien suggestifs du monde bigourdan à Saint-Domingue au XVIIIème siècle. Lacaye est le type du négociant colonial, issu de la bourgeoisie provinciale tarbaise, qui ira dans la grande île faire fructifier les capitaux par le négoce. Il sera aussi propriétaire mais la plantation est ici le résultat non l'origine de la fortune. Le processus a été l'achat d'une maison, d'un commerce, enfin l'achat d'une place qui peu à peu est installée en caféière.

L'ascension sociale de Laporte est différente. Laporte a commencé par être employé dans la boutique du sieur Chatard,

1 Voir notre Revue, VIII: 139-153. - R. Massio, «Les Sources privées de l'histoire coloniale dans le pays de Bigorre (1950-1952) 》, Actes du 78ème Congrès des Sociétés Savantes, Toulouse, 1953, 23-35. (Paris. Presses Universitaires de France, 1954).

2 R. Massio, «Le petit monde Bigourdan de Saint-Domingue au XVIIIème siècle », Annales des Antilles, No 2 (Avril 1955), 66-70. 
apothicaire breveté du roi au Cap. Par la suite, Chatard lui cédera une saline qu'il possédait à Port-à-Piment, ainsi que son fonds d'apothicaire. C'est la fortune pour Laporte, car l'officine vaut deux millions de livres. Nous ne pouvons suivre les étapes de cette « brillante fortune » qui va servir à acquérir la seigneurie de Pouyastruc-en-Bigorre. Laporte est un exemple de réussite parmi les Bigourdans. C'est aussi la preuve qu'à SaintDomingue l'argent se fait par le commerce, par les affaires autant que par la terre et plus vite.

Lettres de Bigourdans à Saint-Domingue au XVIIIème siècle. ${ }^{3}-$

Nous possédons quelques lettres, épaves d'archives familiales, dans lesquelles se trouvent exposés certains aspects généraux de la vie de l'île. Elles ont pour auteur un Bigourdan gérant de plantation, Luro; un Bigourdan établi à Jérémie; enfin un fils de famille noble de Vic-en-Bigorre, Colomés de Juillan.

Dans la missive de Luro, on peut entrevoir le rôle du gérant à Saint-Domingue sur une plantation. Il ne diffère guère d'une habitation à l'autre si ce n'est dans la facilité ou la difficulté de tromper le maître absent. Plus intéressant est dans cette lettre le reflet de la grande peur sociale des blancs en 1789 . Le nègre est déjà considéré comme un ennemi prêt à se révolter.

Avec Villat de Jérémie, nous sommes à un autre tournant de l'histoire de l'île. C'est l'exode, les derniers départs de 1803 vers une Antille voisine dans l'attente de jours meilleurs. On veut encore vivre d'espoir.

Colomés de Juillan est différent. Il apparaît comme l'exemple classique du fils de famille exilé à Saint-Domingue et repenti. Il nous donne des détails sur ses compatriotes bigourdans déjà installés, donc une vue sur l'émigration bigourdane aux îles dont nous avons eu l'occasion de parler et qui complète notre

${ }^{3}$ Ces lettres communiquées par MM. Rivière de Bazillac et Laporte de Lafitole (Hautes-Pyrénées) ont été publiées dans la Revue de la Société Haitienne d'histoire, Vol. 98 (Juillet 1955), 65-75 (cf. R. Massio, Lettres de Bigourdans à Saint-Domingue au XVIIIème siècle). 
dossier. ${ }^{4}$ On voit notamment dans quelles conditions mourut Thèze de Camalès-en-Bigorre. Pour tous nos Bigourdans la période des troubles a succédé à la période heureuse. De plus, Colomés de Juillan décrit la situation au Cap en 1791 à un moment particulièrement critique.

Ainsi ces trois petites lettres, tout en apportant une nouvelle clarté sur le XVIIIème siècle colonial bigourdan, permettent de saisir sur le vif quelques aspects de la vie de Saint-Domingue. Elles présentent un réel intérêt documentaire et nos regrets sont grands de songer que bien d'autres correspondances de cette nature ont pu disparaître.

\section{Les Papiers Carrère..$^{5}$}

Les papiers Carrère sont beaucoup plus importants. Ils constituent un petit dossier de plantation.

Ces papiers sont ceux de François de Carrère, originaire de Cieutat-en-Bigorre et propriétaire de l'habitation qui portait le nom de Beauvallon au Fond Ferrier et de deux immeubles à Port-au-Prince. L'origine et l'histoire de la plantation apparaissent à travers les différents titres de propriété. Nous avons quelques détails sur un quartier caféier, le quartier du Fond Ferrier, à l'extrémité de la plaine de Léogane, dans le fond de la vallée, sur son développement en 1750 , c'est-à-dire au début de la culture en grand du café et alors qu'il n'y a plus de terres libres à prendre dans la plaine de Léogane. C'est la marche des pionniers vers les mornes et combien de concessions durent changer de mains par des moyens plus ou moins honnêtes. Dans ce dossier on peut voir aussi comment étaient utilisés les revenus retirés de la culture, d'abord réemployés en France dans l'achat de terres, puis dans l'achat d'immeubles à Saint-Domingue, au Port-au-Prince. C'est

${ }^{4} \mathrm{R}$. Massio, «Les Bigourdans à Saint-Domingue au XVIIIème siècle 》, Annales du Midi, No 2 (Avril 1952), 151-158, et dans la même Revue: "La Bigorre et Saint-Domingue au XVIIIème siècle», (Janvier 1954), No 1 , 21-46.

5 Ces papiers sont la propriété de M. le Colonel de Carrère, à Tarbes (1, Rue du Quatre Septembre). Nous nous proposons de publier d'après ces documents une étude intitulée: Une famille noble de Bigorre à SaintDomingue au XVIIIème siècle. 
le début de ce qu'on a appelé à juste titre le capitalisme familial et des fortunes bigourdanes ont peut-être eu une origine coloniale. A l'origine de cette richesse, une alliance avec la bourgeoisie commerçante de l'île, déjà installée avant l'arrivée de Carrère. Le mariage n'est-il pas le début de bien des fortunes coloniales ? C'est par son mariage avec une fille Dugué, c'est par une charge de notaire, c'est par l'octroi d'emplacements urbains et de concessions dans les mornes que Carrère, noble bigourdan émigré à Saint-Domingue, va entrer dans le monde des grands propriétaires coloniaux.

Un inventaire après la mort de Carrère, en 1784, permet d'étudier la question de la main-d'œuvre, la situation de la plantation, le cadre dans lequel s'est déroulée l'existence du planteur et quel fut son niveau social. Le monde du commerce, ses difficultés, pourront être évoqués, grâce à quelques lettres de négociants, de même le monde des hommes d'affaires et aussi celui plus modeste de compatriotes bigourdans. Les troubles sociaux et politiques durant la Révolution et la question de l'indemnité si longtemps attendue sont aussi en bonne place dans notre dossier.

Bourgeoisie urbaine ou rurale, petite noblesse, se retrouvent ici. C'est bien l'envers d'un siècle sur lequel tout n'a pas été dit. A Saint-Domingue, planteurs, boutiquiers ou petits blancs montrent que les structures sociales ne sont plus les mêmes qu'en France. L'origine des fortunes, leur processus, l'œuvre des pionniers auprès des mornes, de nombreux problèmes se posent, difficiles si l'on va au fond des choses.

Ajoutons ces nouvelles sources privées de l'histoire coloniale à celles déjà indiquées dans notre précédente chronique et conservons l'espoir d'autres trouvailles.

ROGER MASSIO 